\title{
Occult metastatic thyroid cancer diagnosed during breast cancer axillary sentinel node biopsy
}

\author{
Pratima Herle ${ }^{1} 1,2$, Steven Boyages ${ }^{3}$, Rina Hui4, Najmun Nahar5 and Nicholas K Ngui² \\ 1Department of Surgery, Westmead Hospital, Westmead, New South Wales, Australia, ${ }^{2}$ General Surgery, Mount \\ Druitt Hospital, Mount Druitt, New South Wales, Australia, 3Department of Endocrinology, Westmead Hospital, \\ Westmead, New South Wales, Australia, ${ }^{4}$ Department of Radiation Oncology, Sydney West Cancer Network, Sydney, \\ New South Wales, Australia, and 5Department of Medical Oncology, Sydney West Cancer Network, Sydney, \\ New South Wales, Australia
}

Correspondence should be addressed to $\mathrm{P}$ Herle

Email

pratima.herle@gmail.com

\section{Summary}

In most developed countries, breast carcinoma is the most common malignancy in women and while thyroid cancer is less common, its incidence is almost three to five times greater in women than in men. Since 1966, studies have demonstrated an association between thyroid and breast cancer and despite these studies, the mechanism/s by which they are related, remains unclear. We present a case of a 56-year-old lady who initially presented in 2014 with a screen detected left breast carcinoma but was subsequently found to have occult metastatic thyroid cancer to the axilla, diagnosed from a sentinel node biopsy from the primary breast procedure. The patient underwent a left mastectomy, left axillary dissection and total thyroidectomy followed by three courses of radioactive iodine ablation. Despite this, her thyroglobulin level continued to increase, which was secondary to a metastatic thyroid cancer parasternal metastasis. Breast and thyroid cancer presents metachronously or synchronously more often than by chance. With improving mortality in primary cancers, such as breast and differentiated thyroid cancer, it is likely that as clinicians, we will continue to encounter this association in practice.

\section{Learning points:}

- There has been a long-standing observation of an association between breast and thyroid cancer although the exact mechanism of this association remains unclear.

- Our patient presented with thyroid cancer with an incidental diagnosis from a sentinel node biopsy during her primary breast operation for breast cancer and was also found to have a parasternal distant bony metastasis.

- Thyroid axillary metastases are generally rare.

- The interesting nature in which this patient's metastatic thyroid carcinoma behaved more like a breast carcinoma highlights a correlation between these two cancers.

- With improving mortality in these primary cancers, clinicians are likely to encounter this association in clinical practice.

- Systemic therapy for metastatic breast and thyroid cancers differ and therefore a clear diagnosis of metastasis is crucial. 


\section{Background}

In most developed countries, breast carcinoma is the most common malignancy in women and while thyroid cancer is less common, the incidence of thyroid cancer is almost three to five times greater in women than in men $(1,2)$. The association between breast and thyroid carcinoma has been reported in the literature as early as 1966 (3). Since then, numerous studies have demonstrated that these cancers occur metachronously or synchronously more often than can be attributed to chance $(1,4,5,6)$. Hormonal, genetic and environmental factors have been implicated in explaining this association.

We present a case of a 56-year-old lady who initially presented with a breast cancer but was subsequently found to have occult metastatic thyroid cancer to the axilla, found in an axillary sentinel node. With reference to the current literature, the possible factors contributing to the association between breast and thyroid cancer and also, the common sites of metastases of thyroid cancer is explored.

\section{Case presentation}

We present the case of a 56-year-old female patient who attended Mount Druitt Hospital Surgical Outpatient Clinic (NNg) in November 2014. A routine breast screen mammogram had found cancer in the left breast. She reported also noticing a lump in that breast for several months, but no thyroid or neck symptoms.

She had a history of type 2 diabetes mellitus but no other significant past medical history. She was from a family of 15 siblings, of which one elder sister had passed away from breast cancer at 60 years of age. There was no other significant family history of breast, ovarian or endocrine cancer. The patient had three children, all of whom were breastfed. She was not a smoker and drank alcohol socially.

On examination of the left breast, there was a palpable retroareolar mass approximately $3 \mathrm{~cm}$ in size, causing nipple retraction. Examination of the right breast was unremarkable. Screening mammography, breast ultrasound and core biopsy of this left breast mass confirmed invasive ductal carcinoma. A variety of surgical management options were discussed and the patient proceeded with a total mastectomy and sentinel lymph node biopsy.

Four specimens were sent for pathology from this operation:

(1) Left mastectomy
(2) Left axillary sentinel lymph node, level 1 (intraoperative count 17 134)

(3) Left axillary sentinel lymph node, level 2 (count 626)

(4) Left axillary sentinel lymph node (count 215)

The pathology demonstrated a $32 \mathrm{~mm}$, grade 2 invasive ductal carcinoma with $42 \mathrm{~mm}$ of ductal carcinoma in situ (DCIS), with clear margins, oestrogen (90\% nuclei stained and moderate intensity of staining) and progesterone receptor positive $(80 \%$, strong), HER 2 negative and Ki67 score of $12 \%$ in the left mastectomy specimen. One sentinel lymph node (count 17 134) was positive with a 2.4 $\mathrm{mm}$ breast carcinoma macrometastasis with a confirmed extranodal spread. Incidentally, the axillary sentinel node with the count of 626 demonstrated a metastatic papillary thyroid carcinoma (Fig. 1). The sentinel lymph node with the count of 215 did not contain any abnormal pathology

\section{Investigation}

A thyroid ultrasound was performed and revealed a multilobulated solid mass occupying most of the left lobe thyroid with multiple coalescent nodules $(48 \times 30 \times 25$ $\mathrm{mm}$ ) and the right lobe and cervical nodes were normal (Fig. 2). Fine needle biopsy (FNB) was performed on the left thyroid mass, which revealed a Bethesda 2009 Classification II lesion (benign).

Her case was discussed at the Westmead Breast Cancer Institute Multidisciplinary Team (MDT) meeting, and it was decided to proceed with left axillary lymph node
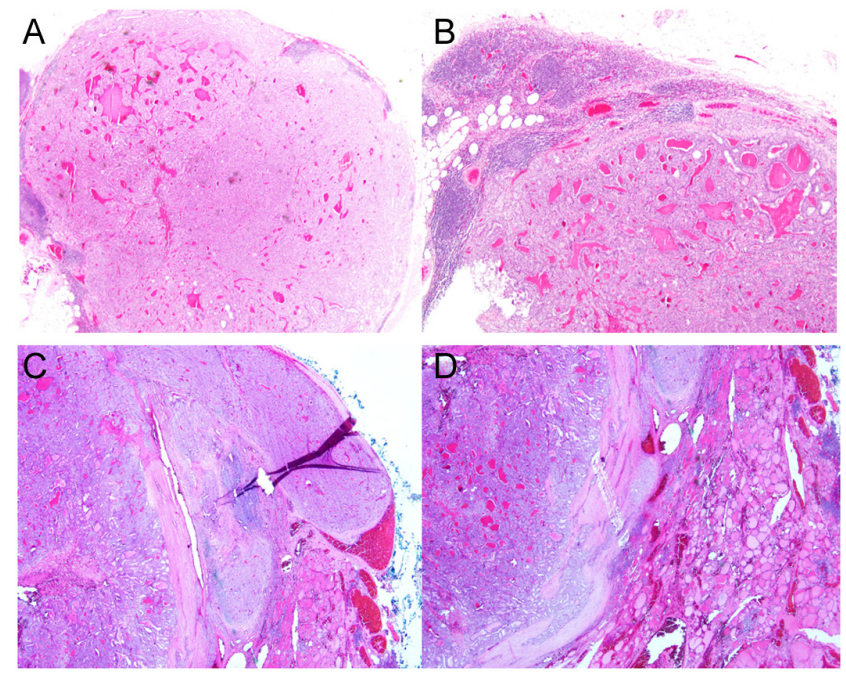

Figure 1

Sentinel lymph node with thyroid cancer and morphology of thyroid cancer in thyroid. (A) Sentinel lymph node containing thyroid cancer (low power). (B) Sentinel lymph node containing thyroid cancer (high power). (C) Thyroid cancer in thyroid (low power). (D) Thyroid cancer (high power). 


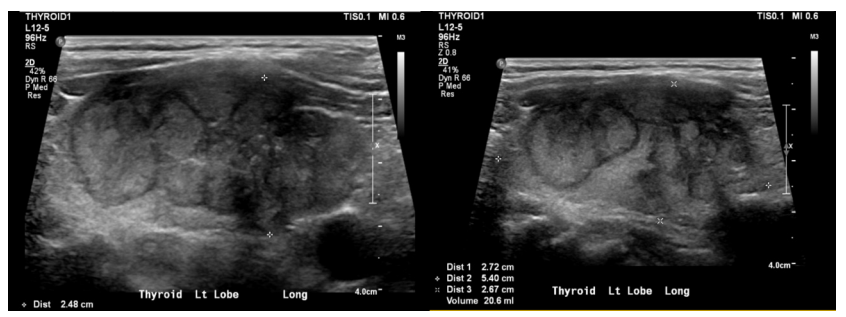

Figure 2

Thyroid ultrasound in December 2014 demonstrating $5 \mathrm{~cm}$ left thyroid mass with multiple coalescent nodules.

clearance with a total thyroidectomy in January 2015. This was then followed by chemotherapy, antioestrogen therapy and radioactive iodine treatment.

Pathology demonstrated a $55 \mathrm{~mm}$ invasive follicular variant of papillary thyroid cancer (BRAF negative) with locally invasive features (lymphovascular invasion, extrathyroidal extension present) with identical appearance to the metastasis present in the axillary sentinel lymph node (Fig. 1). Pathology also demonstrated nil further axillary metastases (0/11 lymph nodes).

\section{Treatment}

The patient then received four cycles of adjuvant chemotherapy (AC (doxorubicin and cyclophosphamide) and Taxol (Paclitaxel)) (RH) followed by anastrozole for a total of 10 years.

Concurrently, she was also managed by an endocrinologist (SB) for radioactive iodine therapy. In October 2015, she received an ablative dose of radioactive iodine (I-131) and post-ablative iodine imaging demonstrated possible residual thyroid tissue in the thyroid bed and focal uptake in the right second intercostal space (Fig. 3). As this was an unusual location for a thyroid cancer metastasis, this mass was agreed to be followed up in subsequent years.

\section{Outcome and follow-up}

On annual review in December 2016, her thyroglobulin (Tg) level was detectable at $1.7 \mu \mathrm{g} / \mathrm{L}$ (all $\mathrm{Tg}$ levels are unstimulated values). Ultrasound of the neck in January 2017 demonstrated possible residual thyroid tissue which was later not visualised on CT and also did not detect any cervical lymphadenopathy. In November 2017, thyroglobulin level remained elevated at $18.5 \mu \mathrm{g} / \mathrm{L}$, and she proceeded to have a second dose of radioactive iodine in February 2018. Once again, post-ablative iodine imaging demonstrated a focal moderately intense focus of
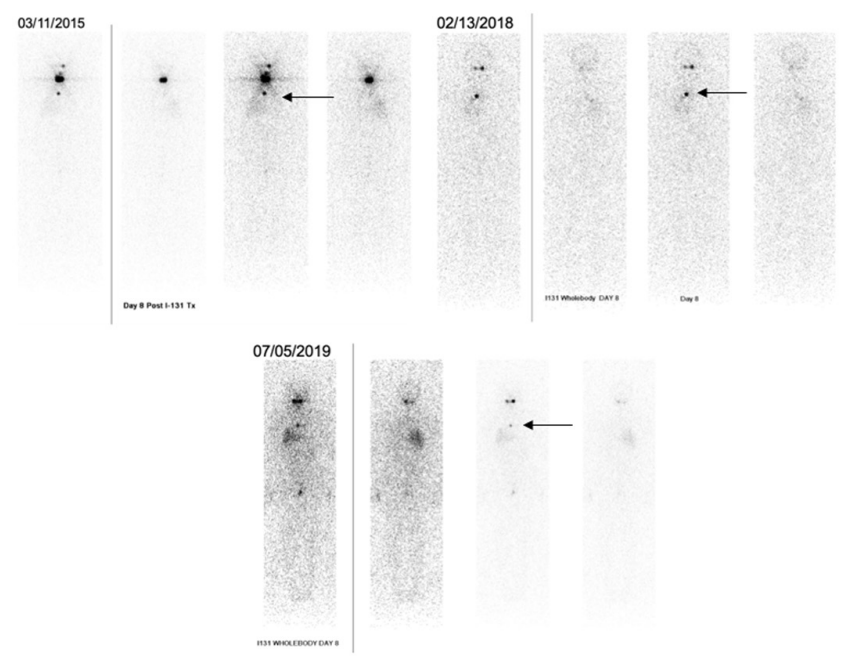

Figure 3

Post-iodine ablation lodine (I-131) whole body imaging in 2015, 2018 and 2019 with arrows demonstrating focal uptake right of the sternal body.

I-131 uptake adjacent to sternum thought to either be a bony metastasis or internal mammary node (Fig. 3).

In November 2018, thyroglobulin level remained detectable at $8 \mu \mathrm{g} / \mathrm{L}$, and this led the patient to have a FDG-PET CT, which demonstrated a small region of mildly increased metabolism on the right-side of the body of the sternum (Fig. 4). A whole body bone scan was also performed and did not demonstrate evidence of any osseous metastasis (Fig. 5). In May 2019, the patient's thyroglobulin level was elevated at $59.6 \mu \mathrm{g} / \mathrm{L}$. She was administered a third dose of radioactive iodine (total radiation dose $3.46 \mathrm{GBq}$ ) and post-radioactive iodine imaging once again demonstrated the focal parasternal activity.

Given the elevated levels of thyroglobulin, lack of avidity on bone scan but I-131 avidity, this right sided parasternal mass was thought to be metastatic thyroid cancer refractory to radioactive iodine. She was subsequently referred to Radiation Oncology ( $\mathrm{NNa}$ ) and has completed two doses of stereotactic body radiotherapy
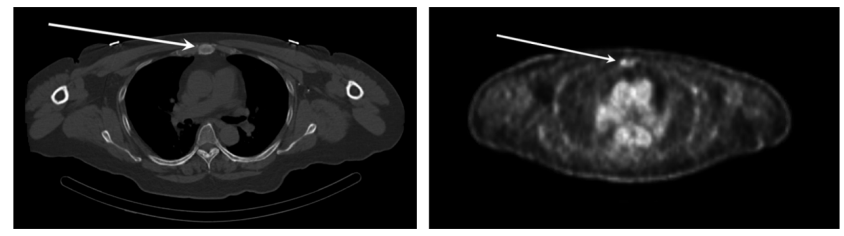

Figure 4

Axial images from CT (left) and FDG-PET CT (right) in 2018. Demonstrating the presence of a lesion at the level of the sterno-manubrial junction. It is also demonstrating increased metabolic uptake on FDG-PET imaging (right). 


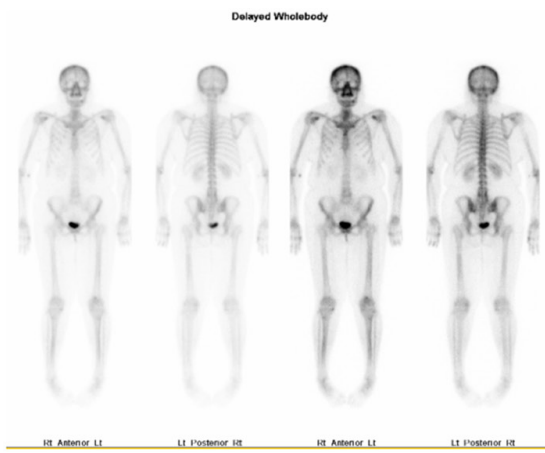

for this lesion in March 2020 and continues to be followed up.

\section{Discussion}

In 1966, Chalstrey and Benjamin suggested a possible association between thyroid and breast cancer. They followed 92 female patients with thyroid cancer over a 20-year period (1945-1964) and noted that $8.7 \%$ of these patients also developed breast carcinoma, a higher incidence than in comparable age groups at the time. Since then, several studies have supported this association. Joseph et al. (2) performed a meta-analysis of a total of 18 studies and demonstrated that there was a significantly increased risk of developing thyroid cancer after a primary diagnosis of breast cancer (BC $\rightarrow$ TC) (standardised incidence ratio $(\mathrm{SIR})=1.59,95 \% \mathrm{CI}: 1.28-1.99, P<0.01)$. Similarly, there was an increased risk of developing breast cancer as a second primary malignancy of thyroid cancer $(\mathrm{TC} \rightarrow \mathrm{BC}) \quad(\mathrm{SIR}=1.24,95 \% \mathrm{CI}: 1.16-1.33, P=0.005)$. Despite several studies demonstrating an association between breast and thyroid cancer, the mechanism/s in which they are related remains elusive. Common aetiologies between the two cancers include hormonal, genetic, environmental and therapeutic factors.

One possible relationship is that both the breast and the thyroid are glands regulated by the hypothalamicpituitary axis (1). The role of sex hormones have been well studied and defined in breast cancer and thyroid cancer demonstrate a gender disparity favouring women. Sex hormones are a major difference between men and women, raising the possibility that this is a common factor between breast and thyroid cancer. Studies have demonstrated that the expression of oestrogen receptors (ER) and progesterone receptors (PR) have been significantly higher in breast cancer patients with co-existing thyroid cancer, compared to those without (4, 7). Supporting this, our patient's breast cancer pathology also demonstrated that her tissue was ER (90\%) and

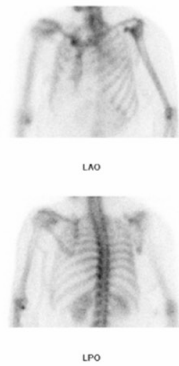

Figure 5

Whole body bone scan (April 2019).

Demonstrating no evidence of increased uptake in skeleton.
PR (80\%) positive. Furthermore, previous studies have demonstrated that sex steroid receptors were not only found in human thyroid tissue but in also thyroid cancer cell lines and the levels of ER were significantly higher in thyroid cancer compared with normal thyroid tissue (1). Thyroid hormone also exerts multiple biological effects in almost all human tissue, including the mammary gland, however, its role in breast cancer is uncertain (1).

Other potential common aetiological factors are postulated to be therapeutically related to the primary malignancy diagnosed. Previously, the use of radioactive iodine in thyroid cancer was thought to increase the risk of breast cancer but further studies demonstrated an increased risk of breast cancer following thyroid cancer regardless of radioactive iodine administration (1). Our patient's presentation with simultaneous breast and metastatic thyroid cancer without having undergone either radioactive iodine therapy or radiotherapy to her breast, possibly negates these suggestions.

Genetics has also been suggested as a possible aetiological factor explaining the association between breast and thyroid cancer. Patients with Cowden syndrome have alterations in the PTEN gene, and these patients have higher rates of developing breast and thyroid cancers (2). Familial cancers have more of a tendency to appear at younger ages than sporadic cases. Young patients with second primary malignancy of BC or TC should be studied for a genetic association between the two cancers.

Environmental factors such as high BMI have demonstrated a positive association between breast and thyroid cancer independently but this is a weak association (1). Several studies also suggest an element of detection or screening bias leading to possible earlier detection of a second malignancy after the diagnosis of the primary malignancy $(5,7,8)$. Our patient's incidental finding of metastatic thyroid cancer to the axilla during the primary breast cancer surgery suggests that the association between thyroid and breast cancer exists beyond screening bias alone. 
It is unusual that pre-operative FNB of our patient's thyroid nodule yielded a benign result. Larger thyroid nodules $(>3 \mathrm{~cm})$ could have higher false-negative rates $(\sim 3 \%)$ due to larger amounts of background colloid mistaken for benign adenomatous colloid. General recommendations are to consider clinical suspicion, size and suspicious radiological features/TIRADS classification to facilitate surgical decision making (9).

Another highlight from our case is the unusual nature of distant metastases from the patient's differentiated thyroid cancer. Not only was her thyroid cancer diagnosed from the axillary sentinel lymph node biopsy from her primary breast operation but over the course of 5 years, she was noted to likely have a parasternal bony distant metastasis. While some case reports are available, axillary metastases from differentiated thyroid cancer are rare, especially without the involvement of local cervical nodes (10). Common sites of distant metastasis of differentiated thyroid cancer are lung and bone and axillary metastases are extremely rare (11). The patient's parasternal mass (first diagnosed on radioactive I-131 imaging postablation in 2015) was persistently noted on follow up imaging. As this location was more common for breast cancer metastasis, this presented a diagnostic dilemma at the time. It was only due to persistently elevated levels of thyroglobulin, lack of avidity on bone scan but I-131 avidity that this mass was thought secondary to thyroid cancer and hence, was elected to treat with stereotactic body radiotherapy.

Despite their association, systemic therapy for metastatic breast and thyroid cancers differ and therefore a clear diagnosis of metastases is crucial. If there are new metastatic sites and investigations with serum thyroglobulin levels and nuclear bone scans are not helpful, a tissue biopsy may be necessary. CDK4/6 inhibitor, PI3K inhibitor or mTOR inhibitor are effective targeted therapies for hormone receptor positive, HER2 negative, advanced breast cancer. To date, there is only preclinical data suggesting a potential rationale for the use of these inhibitors in aggressive thyroid cancers (12). Lenvatinib, a multi-kinase inhibitor targeting VEGFR1-3, FGFR1-4, PDGFR, RET and KIT is an effective treatment for metastatic thyroid cancer and could be used if there is further metastatic spread or the thyroid cancer deposit fails to respond (13). Although preliminary clinical data suggested a possible role in targeting FGFR1 in some patients with hormone receptor positive, HER2 negative, advanced breast cancer, this type of targeted therapy is still in early clinical development and is not standard of care (14).
Our patient's presentation reinforces the already known association between breast and thyroid cancer but further research is still needed to explain this association. In clinical practice, it would be important to bear this in mind during initial presentation or follow up of a patient with known primary breast or thyroid cancer.

\section{Declaration of interest}

The authors declare that there is no conflict of interest that could be perceived as prejudicing the impartiality of the research reported.

\section{Funding}

This research did not receive any specific grant from any funding agency in the public, commercial or not-for-profit sector.

\section{Patient consent}

Publication of the case report including photographs of relevant imaging and pathology has been discussed with the patient and informed consent has been obtained.

\section{Author contribution statement}

$\mathrm{PH}$ is the primary author, involved in collating data and information, preparation of report. S B is the patient's treating Endocrinologist, supervisor and helped in editing the manuscript. $\mathrm{N} \mathrm{Na}$ is the patient's treating Radiation Oncologist, supervisor and was involved in editing the manuscript. $\mathrm{R} \mathrm{H}$ is the patient's treating Medical Oncologist and supervisor. $\mathrm{N} \mathrm{Ng}$ is the patient's treating Surgeon, primary clinical supervisor and involved in conceptualisation of report and helped in editing the manuscript.

\section{References}

1 Dong L, Lu J, Zhao B, Wang W \& Zhao Y. Review of the possible association between thyroid and breast carcinoma. World Journal of Surgical Oncology 201816 130. (https://doi.org/10.1186/s12957-0181436-0)

2 Joseph KR, Edirimanne S \& Eslick GD. The association between breast cancer and thyroid cancer: a meta-analysis. Breast Cancer Research and Treatment 2015152 173-181. (https://doi.org/10.1007/ s10549-015-3456-6)

3 Chalstrey LJ \& Benjamin B. High incidence of breast cancer in thyroid cancer patients. British Journal of Cancer 196620 670-675. (https://doi.org/10.1038/bjc.1966.78)

4 Zhang L, Wu Y, Liu F, Fu L \& Tong Z. Characteristics and survival of patients with metachronous or synchronous double primary malignancies: breast and thyroid cancer. Oncotarget 20167 52450-52459. (https://doi.org/10.18632/oncotarget.9547)

5 Chen AY, Levy L, Goepfert H, Brown BW, Spitz MR \& Vassilopoulou-Sellin R. The development of breast carcinoma in women with thyroid carcinoma. Cancer 200192 225-231. (https:// doi.org/10.1002/1097-0142(20010715)92:23.0.CO;2-B)

6 Garner CN, Ganetzky R, Brainard J, Hammel JP, Berber E, Siperstein AE \& Milas M. Increased prevalence of breast cancer among patients with thyroid and parathyroid disease. Surgery 2007 
142 806-813; discussion 813.e1-3. (https://doi.org/10.1016/j. surg.2007.07.024)

7 An JH, Hwangbo Y, Ahn HY, Keam B, Lee KE, Han W, Park DJ, Park IA, Noh DY, Youn YK, et al. A possible association between thyroid cancer and breast cancer. Thyroid 201525 1330-1338. (https://doi.org/10.1089/thy.2014.0561)

8 Huang Ns, Chen Xx, Wei Wj, Mo M, Chen Jy, Ma B, Yang Sw, $\mathrm{Xu} \mathrm{Wb}, \mathrm{Wu}$ J, Ji Qh, et al. Association between breast cancer and thyroid cancer: a study based on 13978 patients with breast cancer. Cancer Medicine 20187 6393-6400. (https://doi.org/10.1002/ cam4.1856)

9 Nam SJ, Kwak JY, Moon HJ, Yoon JH, Kim EK \& Koo JS. Large ( $\geq 3$ $\mathrm{cm})$ thyroid nodules with benign cytology: can thyroid imaging reporting and data system (TIRADS) help predict false-negative cytology? PLoS ONE 201712 e0186242. (https://doi.org/10.1371/ journal.pone.0186242)

10 Hafez MT, Refky B, Abd Elwahab K, Arafa M, Abdou I \& Elnahas W. Axillary lymph nodes metastasis in a patient with recurrent papillary thyroid cancer: a case report. Journal of Medical Case Reports 20159 $1-3$.

11 Chen D, Huang L, Chen S, Huang Y, Hu D, Zeng W, Wang M, Zhou W, Feng H, Wei W, et al. Innovative analysis of distant metastasis in differentiated thyroid cancer. Oncology Letters 202019 1985-1992. (https://doi.org/10.3892/ol.2020.11304)

12 Cabanillas ME, Ryder M \& Jimenez C. Targeted therapy for advanced thyroid cancer: kinase inhibitors and beyond. Endocrine Reviews 2019 40 1573-1604. (https://doi.org/10.1210/er.2019-00007)

13 Schlumberger M, Tahara M, Wirth LJ, Robinson B, Brose MS, Elisei R, Habra MA, Newbold K, Shah MH, Hoff AO, et al. Lenvatinib versus placebo in radioiodine-refractory thyroid cancer. New England Journal of Medicine 2015372 621-630. (https://doi.org/10.1056/NEJMoa1406470)

14 Hui R, Pearson A, Cortes J, Campbell C, Poirot C, Azim HA, Fumagalli D, Lambertini M, Daly F, Arahmani A, et al. Lucitanib for the treatment of HR+/HER2- metastatic breast cancer: results from the multicohort Phase II FINESSE study. Clinical Cancer Research 2020 26 354-363. (https://doi.org/10.1158/1078-0432.CCR-19-1164)

Received in final form 30 June 2020

Accepted 29 July 2020 